\title{
EFFECT OF LUMBAR SPINE MANIPULATION ON MENSTRUAL DISTRESS
}

Reshma Gurav *1, Shweta Nahar ${ }^{2}$.

${ }^{*}$ Associate Professor, MGM College of Physiotherapy, Kamothe, Navi Mumbai, Maharashtra, India.

${ }^{2}$ Physiotherapist, MPT Musculoskeletal Sciences, MGM Department of Physiotherapy, Kamothe, Navi Mumbai, Maharashtra, India.

\section{ABSTRACT}

Background: Menstrual distress is characterized by crampy pelvic pain beginning before or at onset of menstruation, abdominal pain, bloating, headache, backache, irritability, depression, anxiety, mood changes, fatigue and sleep disturbances. Primary dysmenorrhea is defined as recurrent, painful menses in women in the absence of an identifiable pelvic pathology. Secondary dysmenorrhea is menstrual pain associated with underlying pelvic pathology. The aim of the study was to find the efficacy of lumbar spine manipulation on menstrual distress.

Methods: The study was a cross-sectional study. Menstrual Distress Questionnaire (MDQ) and Numerical Pain Rating Scale (NRS) were used as an outcome measure. The lumbar spine manipulation technique was given to the participants.

Results: The post lumbar manipulation technique showed the significant improvement in menstrual distress symptoms along with significant improvement in backache, headache, abdominal discomfort and work absenteeism.

Conclusion: The lumbar spine manipulation is effective in relieving menstrual distress symptoms.

KEY WORDS: Lumbar spine manipulation, Menstrual distress, Menstrual Distress Questionnaire, Dysmenorrhea.

Address for correspondence: Dr. Reshma S. Gurav (PT), Associate Professor, Department of Musculoskeletal Sciences, MGM College of Physiotherapy, Kamothe, Navi Mumbai, Maharashtra, India. Mobile No.9920332810 E-Mail: reshmagurav81@gmail.com

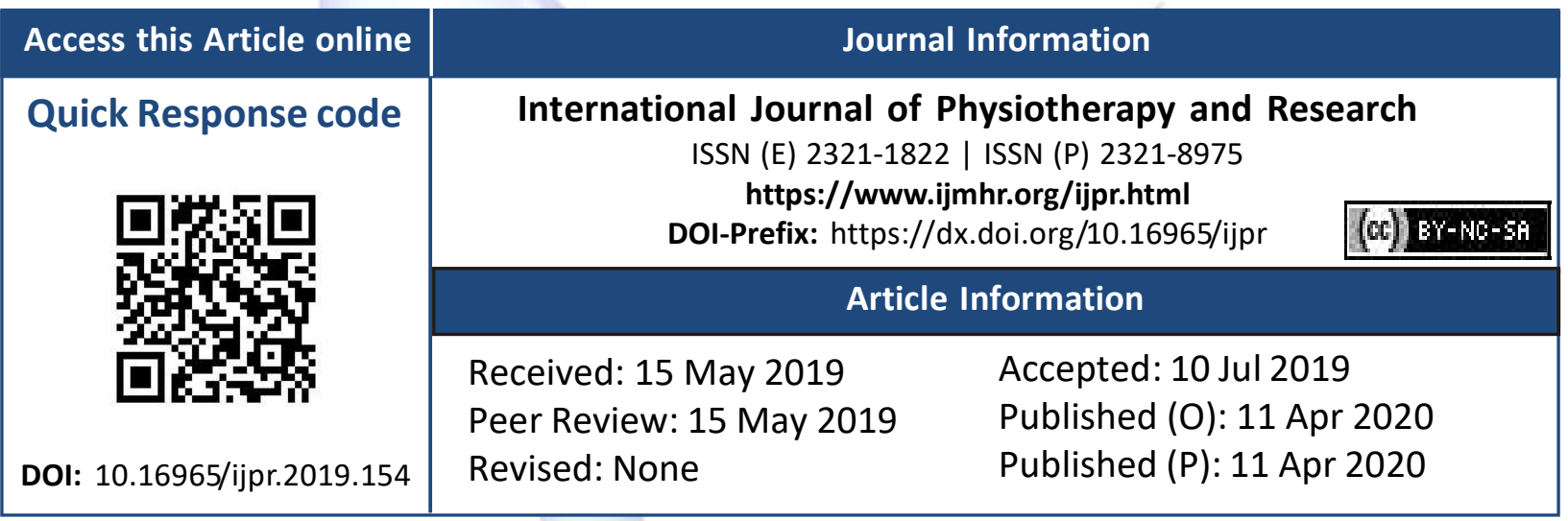

\section{BACKGROUND}

Menstrual distress is characterized by crampy pelvic pain beginning before or at onset of menstruation, abdominal pain, bloating, headache, backache, irritability, depression, anxiety, mood changes, fatigue and sleep disturbances. In very severe forms, it is considered a depressive mood disorder [1]. Dysmenorrhea can be divided into two broad categories of primary and secondary. Primary dysmenorrhea is defined as recurrent, painful menses in women in the absence of an identifiable pelvic pathology.
Secondary dysmenorrhea is menstrual pain associated with underlying pelvic pathology such as endometriosis. Primary dysmenorrhea usually begins in adolescence after the establishment of ovulatory cycles [2].

Primary dysmenorrhea is a gynaecological medical condition of pain during menstruation that interferes with daily activities, as defined by ACOG [3]. It is also defined as painful menstruation of sufficient magnitude so as to incapacitate day-to-day activities [4]. 
$90 \%$ of women presenting suffer from some menstrual pain [5]. Symptoms are frequently associated with time lost from school, work, or other activities [6].

The most widely used drugs for the relief of pain of primary dysmenorrhea are the NSAIDS (ibuprofen, naproxen and mefenamic acid) which act by inhibiting prostaglandin synthetase, a collective name for enzymes in the cyclo-oxygenase pathway of arachidonic acid metabolism to prostaglandins. NSAIDS are effective in relieving pain in $70-85 \%$ of women with confirmed primary dysmenorrhea. Oral Contraceptives $(\mathrm{OC})$ are also used in treatment of primary dysmenorrhea. OCs reduce the levels of prostaglandins in menstrual fluid by blocking ovulation and perhaps, in the case of gestagendominated OCs by reducing uterine sensitivity to vasopressin and PGF ${ }_{2 \mathrm{a}}$. Both NSAIDS and OCs however have side effects and their use is not without some risk. NSAIDS may cause gastrointestinal disturbances, nausea, vomiting, constipation, headache, vertigo, fatigue, and allergic reactions. OCs may cause adverse effects on the liver, diminished glucose tolerance and hypertension. Therefore, investigation of alternative nondrug therapy is warranted [7].

Surgical interruption of the sympathetic pelvic nerve pathways is also used as treatment to diminish uterine pain [8]. The efficacy of conventional treatments such as NSAIDs is considerable however the failure rate can still be as high as $20 \%$ to $25 \%$, and there are also a number of associated adverse effects [9]. Natural treatments vary from several different types of chiropractic adjustments, herbal remedies, lifestyle changes (exercise) and diet related changes to name a few [10]. Many women seek alternatives to conventional medical treatments.

Studies suggest that, $30 \%$ to $50 \%$ of the adult population in industrialized nations use some form of complementary therapy to prevent or treat health-related problems. Physical or manipulative treatments have some of the highest rates of physician referral and practice among the many forms of complementary therapy [11]. One popular nondrug treatment modality is spinal manipulation therapy. Spinal manipulation consists of a high velocity thrust applied to a diarthrodial joint over short amplitude [12].
It has been suggested that manipulation of the vertebrae may increase spinal mobility thus improving pelvic blood supply and facilitating pain relief [13].

The aim of the study was to find the efficacy of lumbar spine manipulation on menstrual distress. Study objectives were to find the effectiveness of lumbar spine manipulation using Menstrual Distress Questionnaire (MDQ), to find improvement in backache, abdominal discomfort and headache on NRS scale and to find effectiveness of lumbar manipulation on work absenteeism due to menstrual discomfort using MDQ.

\section{SUBJECTS AND METHODS}

Ethical approval: The Institutional Research Review Committee had approved the study prior to its commencement. Written and signed informed consent was obtained from all the participants.

Study design: The study was a cross-sectional study. 35 females in the age group of 18 to 40 years suffering from primary dysmenorrhea were selected. Females with recent pregnancy, intrauterine device, recent delivery, any pelvic inflammatory diseases, recent lumbar fractures, recent spinal surgeries, mental illness and females suffering from depression were excluded from the study.

Outcome measure: Menstrual Distress Questionnaire (MDQ) and Numerical Pain Rating Scale (NRS) were used in the study.

Menstrual Distress Questionnaire: The Menstrual Distress Questionnaire (MDQ) is a standard method for measuring cyclical perimenstrual symptoms. ${ }^{[13]}$ The MDQ is a $47-$ item self-report inventory for use in the assessment and treatment of premenstrual and menstrual symptoms. The questionnaire contains description of symptoms classified in to eight categories (total 47 items) pain (6), concentration (9), behavioural change (5), autonomic reaction (4), water retention (4), negative effect (8), arousal (5) and control (6).

The MDQ "Form C" comprised of three separate self reporting columns to measure common symptoms and feelings associated with premenstrual, menstrual and post menstrual phases. 
It allows participants to describe their experience and rate the intensity of their experience as follows: $0=$ No experience of symptoms; $1=$ Present, mild; $2=$ Present, moderate; $3=$ Present, strong; 4= Present, severe.

Scoring and interpretation: Each item had five options categorized on a $0-4$ rating scale with scores as mentioned below. The total score was 188. The level of menstrual distress was ranged as follows: 0 is No experience of symptoms, 147 is Mild, 48-94 is Moderate, 95-144 is Strong and $145-188$ is Severe.

Numerical Pain Rating Scale: The Numerical Pain Rating Scale (NRS) is a unidimensional measure of pain intensity. The 11-point numeric scale ranges from ' 0 ' representing one pain extreme (no pain) to ' 10 ' representing the other pain extreme (worst pain imaginable). The respondent is asked to indicate the numeric value on the segmented scale that best describe their pain intensity. Score range from 0-10 points, with higher scores indicating greater pain intensity.

Procedure: 35 female participants were selected for the study based on inclusion criteria. Informed consent was obtained from all the participants. The study objectives were explained to them thoroughly. The Menstrual Distress Questionnaire was filled by participants. According to their last menstrual date provided in MDQ, the luteal phase of individual participant was calculated. Participants were called according to their given dates. Lumbar spine manipulation was delivered bilaterally. Following each manipulation, the subjects were called in their luteal phase. This was done for three consecutive menstrual cycles, with each participant being treated a total of six times. On the last appointment participants filled the Menstrual Distress Questionnaire again.

\section{Lumbar Spine Manipulation Technique (Fig-} ure 1): The patient lies in side-lying position. The therapist stands in front of the patient. The therapist then flexes patient's top leg until there is movement detected at the selected segmental interspinous space and places the patient's foot in the popliteal fossa of the bottom leg. The therapist grasps patient's bottom shoulder and arm and introduced trunk side bending towards the table and contralateral rotation until motion is felt at the specified interspinous space. The patient's positional setup is maintained, while the patient is rolled towards the therapist. Finally, the therapist's arm and body are used to apply a high-velocity, low-amplitude thrust of the pelvis in an anterior direction.

Fig. 1: Lumbar Spine Manipulation.

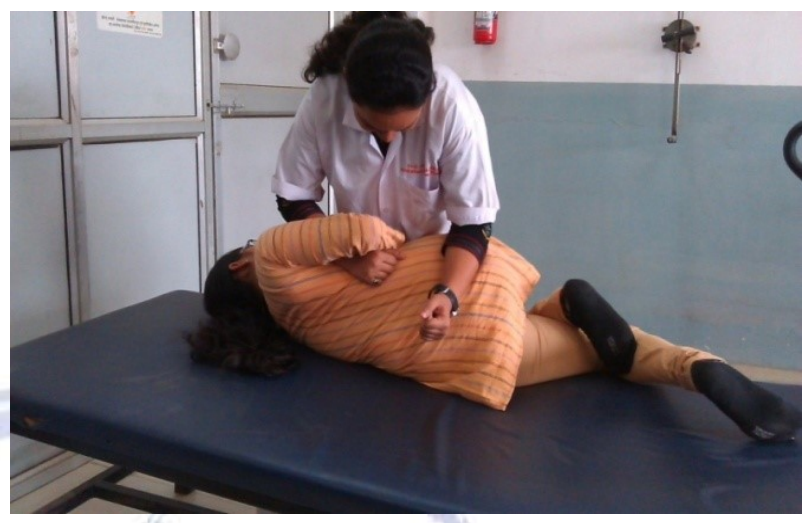

Statistical analysis: Data was analyzed using Statistical Package for Social Sciences (SPSS) software version 16 . The Students paired t-test was used to see the difference in MDQ scores in pre and post lumbar manipulation.

\section{RESULT}

Figure 2 represents the mean score of Menstrual Distress Questionnaire. The difference between mean value of pre and post manipulation on MDQ is 22.87 which is statistically significant $(p$-value< 0.001). (Table 1$)$

Fig. 2: Mean score of Menstrual Distress Questionnaire.

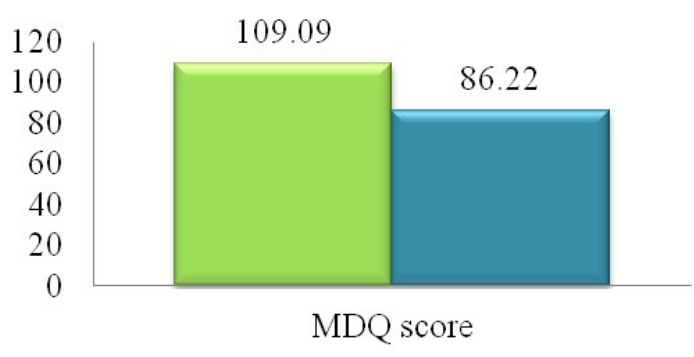

$\square$ Pre-manipulation $\square$ Post-manipulation

Table 1: Mean score of MDQ on pre and post manipulation.

\begin{tabular}{|c|c|c|c|}
\hline & Mean value & Difference & p-value \\
\hline Pre-manipulation & 109.09 & \multirow{2}{*}{22.87} & \multirow{2}{*}{0} \\
\cline { 1 - 2 } Post-manipulation & 86.22 & & \\
\hline
\end{tabular}


Figure 3 represents the mean value difference between pre and post manipulation on NRS for backache is 2.6, headache is 1.51 and abdominal discomfort is 2.77 which are statistically significant. (Table 2)

Fig. 3: Mean score difference of NRS on pre and post manipulation.

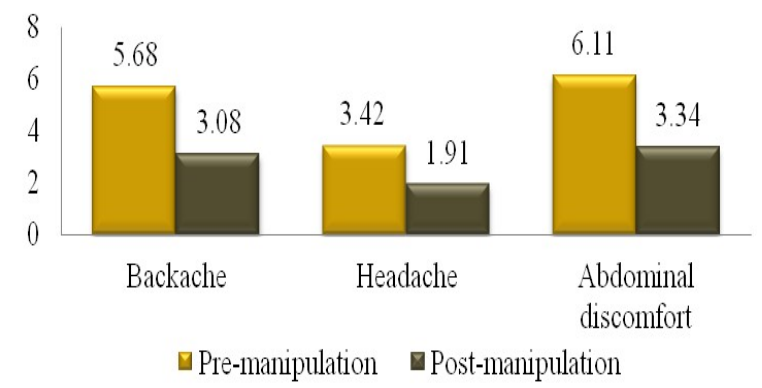

Fig. 3: Mean score difference of NRS on pre and post manipulation.

\begin{tabular}{|c|c|c|c|c|}
\hline & $\begin{array}{c}\text { Pre- } \\
\text { manipulation }\end{array}$ & $\begin{array}{c}\text { Post- } \\
\text { manipulation }\end{array}$ & Difference & p-value \\
\hline Backache & 5.68 & 3.08 & 2.6 & 0 \\
\hline Headache & 3.42 & 1.91 & 1.51 & 0 \\
\hline Abdominal discomfort & 6.11 & 3.34 & 2.77 & 0 \\
\hline
\end{tabular}

Figure 4 represents the mean value difference of overall menstrual discomfort on NRS is 3.95 which is statistically significant. (Table 3 )

Fig. 4: Mean value of overall menstrual discomfort using NRS.

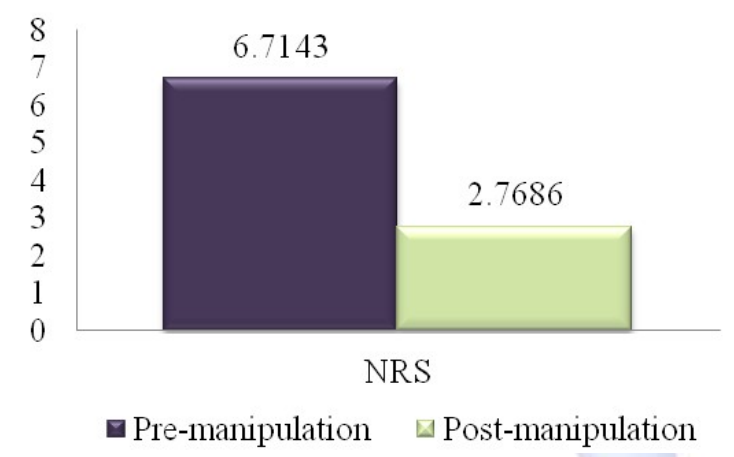

Table 3: Mean score difference of menstrual discomfort on NRS of pre and post manipulation.

\begin{tabular}{|c|c|c|c|}
\hline & Mean value & Difference & p-value \\
\hline Pre-manipulation & 6.71 & \multirow{2}{*}{3.95} & 0 \\
\cline { 1 - 2 } Post-manipulation & 2.76 & & \\
\hline
\end{tabular}

Figure 5 represents the mean value difference between pre and post manipulation for work absenteeism is 0.8 which is statistically significant. (Table 4)
Fig. 5: Mean value of work absenteeism.

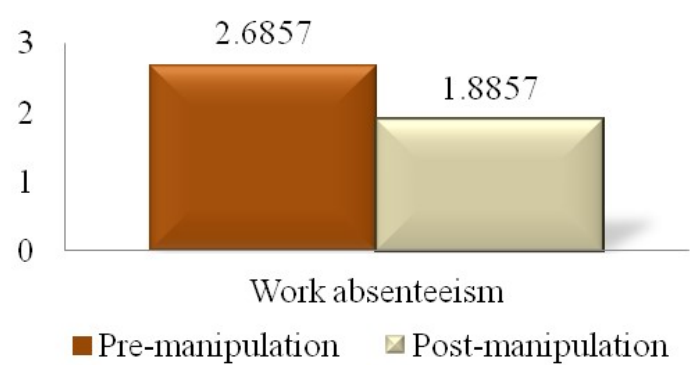

Table 4: Mean score difference of work absenteeism on pre and post manipulation.

\begin{tabular}{|c|c|c|c|}
\hline & Mean value & Difference & p-value \\
\hline Pre-manipulation & 2.68 & \multirow{2}{*}{0.8} & \multirow{2}{*}{0} \\
\hline Post-manipulation & 1.88 & & \\
\hline
\end{tabular}

\section{DISCUSSION}

The result of the study shows improvement in the mean values of backache, headache and abdominal discomfort on NRS scale. The innervations of the uterus arise from the inferior hypo gastric plexus, predominantly from the part of the plexus known as the utero-vaginal plexus. Preganglionic sympathetic fibers of this plexus arise from spinal cord segments $T_{10}-L_{2}$ with parasympathetic preganglionic fibers from sacral segments $S_{2}, S_{3}$ and $\mathrm{S}_{4}$. The major visceral afferent input to the cord is closely related to efferent output and enters the spinal cord at the same levels. Given the above sensory distribution, referred pain from the uterus might be anticipated to involve any somatic areas innervated by the above cord segments. Parasympathetic pelvic nerve pathways are closely associated with spinal vertebrae particularly $\mathrm{S}_{2}-\mathrm{S}_{4}$ segments and $\mathrm{T}_{10}{ }^{-}$ $T_{12}$ segments. Mechanical dysfunction results in reduced spinal mobility due to entrapment of synovial fluid, meniscoids and adhesions affecting the sympathetic nerve supply to blood vessels supplying pelvic viscera leading to dysmenorrhea. Lumbar spine manipulation results in removing the adhesions, meniscoids and stimulation of the parasympathetic area in the spinal cord which in turn results increase in pelvic blood supply thus reducing hypoxia and cramps [14].

Lumbar Spine Manipulation technique is a high velocity thrust is applied to a diarthrodial synovial joint over very short amplitude. This type of manipulation is usually associated with 
an audible "crack," which is often viewed as signifying a successful manipulation. The cracking sound is caused by an event termed "cavitation," occurring within the synovial fluid of the joint. Cavitation is the term used to describe the formation and activity of bubbles (or cavities) within fluid through local reduction in pressure. Spinal manipulations can relieve back pain by taking pressure off sensitive nerves or tissue, increase range of motion, restoring blood flow, reducing muscle tension, and, like more active exercise, promote the release of endorphins within the body to act as natural painkillers $[15,16]$.

Numerous studies show that spinal manipulation increases pain tolerance and its threshold. One mechanism underlying the effects of spinal manipulation is the manipulation's ability to alter central sensory processing by removing subthreshold mechanical or chemical stimuli from paraspinal tissues. Spinal manipulation is also thought to affect reflex neural outputs to both muscle and visceral organs. Substantial evidence demonstrates that spinal manipulation evokes paraspinal muscle reflexes and alters motoneuron excitability $[17,18]$.

Results have shown improvement in Menstrual Distress Questionnaire score after manipulation. The areas where most change was seen were experience with abdominal discomfort and backache few days before the occurrence of menstruation, backache and cramps, aching lower back, abdomen and inner thighs on first day of menstruation. These areas of discomfort saw the greatest positive improvement during the course of study. As the menstrual discomfort has decreased, these have resulted in improvement of daily functions and consequently decrease in absenteeism from school, work and daily scheduled activities.

\section{CONCLUSION}

Menstrual pain associated with primary dysmenorrhea may be alleviated with treatment of motion segment restrictions of the lumbosacral spine with lumbar spine manipulations.

\section{ABBREVIATIONS}

MDQ - Menstrual Distress Questionnaire

NRS - Numerical Pain Rating Scale

OC - Oral Contraceptives

SPSS - Statistical Package for Social Sciences

\section{ACKNOWLEDGEMENTS}

We thank all the participants of the study.

\section{Conflicts of interest: None}

\section{REFERENCES}

[1]. Walsh P, Maxwell J. Premenstrual Syndrome: A Clinical Update for Chiropractor. Chiropractic Journal of Australia, 1993;23:2.

[2]. Antao V, Black A, Burnett M. Primary Dysmenorrhea Consensus Guideline. SOGC Clinical Practice Guideline, Dec 2005;169:1117.

[3]. American Congress of Obstetricians and Gynecologists. 2000.

[4]. Dutta D C. Textbook of gynecology. Sixth edition; 2004. Chapter 2; pg. no. 174-180.

[5]. Jamieson DJ, Stage JF. The Prevalence of Dysmenorrhea, Dyspareunia, Pelvic Pain and Irritable Bowel Syndrome in Primary Care Practices.ObstetGynecol, 1996; 87(1): 55-8.

[6]. Hillen TI, Grbava SL. Primary Dysmenorrhea in Young Western Australian Women: Prevalence, Impact, and Knowledge of Treatment. J Adolesc Health, 1999; 25(1):40-5.

[7]. Kokjohn K, Schmid DM, Triano JJ, Brennan PC. The Effect of Spinal Manipulation on Pain and Prostaglandin Levels In Women with Primary Dysmenorrhea.Journal ofManipulative and Physiological Therapeutics,1992; 15(5): 279-85.

[8]. Perez J.Laparoscopic Presacral Neurectomy: Results of the first 25 cases.Journal of Reproductive Medicine,1990; 35(6): 625-30.

[9]. Dawood M Y. Dysmenorrhea: Pain and Analgesia.1985; 1:20.

[10]. Daley A. The Role of Exercises in Treatment of Menstrual Disorders: The Evidence. British Journal of Gynecology Practice, 2009.

[11]. Astin JA.Why Patients Use Alternative Medicine: Results of a national study.JAMA,1998; 279(19):154853.

[12]. Evans D. Mechanisms And Effects of Spinal Highvelocity, Low-amplitude Thrust Manipulation. JMPT, 2002.

[13]. Moos RH. The development of a menstrual distress questionnaire. Psychosom Med, 1968; 30(6): 85367.

[14]. Hitchcock ME. The manipulative approach to the management of primary dysmenorrhea. The Journal of the American Osteopathic Association,1976; 75(10):909-18.

[15]. Schmidt K., Schweitzer Ali, Tripp J. The Effects of Spinal Manipulation on Menstrual Distress: A Pilot Study. Man Ther. 2010; 40.

[16]. Proctor M, Hing W, Johnson TC. Spinal Manipulation for Dysmenorrhea (Review).The Cochrane Library, 2010; 1.

[17]. Sidana R, Narkeesh M. Effect of Spinal Manipulation in Primary Dysmenorrhea. IJPOT, 2012; 6(2): 101-105.

[18]. Pickar J. Neurophysiological effects of spinal manipulation. The Spine Journal, 2002; 2:357-371. 